\title{
THE MIXING ADVANTAGE IS LESS THAN 2
}

\author{
K. HAMZA, P. JAGERS, A. SUDBURY, AND D. TOKAREV
}

\begin{abstract}
Corresponding to $n$ independent non-negative random variables $X_{1}, \ldots, X_{n}$, are values $M_{1}, \ldots, M_{n}$, where each $M_{i}$ is the expected value of the maximum of $n$ independent copies of $X_{i}$. We obtain an upper bound to the expected value of the maximum of $X_{1}, \ldots, X_{n}$ in terms of $M_{1}, \ldots, M_{n}$. This inequality is sharp in the sense that the quantity and its bound can be made as close to each other as we want. We also present related comparison results.
\end{abstract}

\section{INTRODUCTION}

To illustrate the main thrust of this paper, we consider a simple two-component parallel system; say a light pole made up of two light bulbs. If the system is considered to have failed once both components, assumed to act independently, fail, then a reasonable measure of the performance of the system is $\mathbb{E}[\max (X, Y)]$, where $X$ and $Y$ are the independent random lifetimes of the two components.

Assume that, to build the system, we may choose from any of two manufacturers (i.e. two lifetime distributions). Should we choose two components from the same manufacturer or should we mix? In the case of manufacturers with identical performances $\left(\mathbb{E}\left[\max \left(X_{1}, X_{2}\right)\right]=\mathbb{E}\left[\max \left(Y_{1}, Y_{2}\right)\right]\right)$, there is (almost) always a net gain in mixing. It is then natural to ask how much gain can one achieve and further to identify situations in which this gain is attained.

In the spirit of the above example, we shall call a family of $n$ independent random variables an $n$-assembly. When these are also identically distributed, we will say that they form a similar $n$-assembly. We call performance of an $n$-assembly (whether similar or not) the expected value of its maximum. The aim of this paper is to bound (from above as well as below) the performance of an $n$-assembly relying solely on the performances of all similar $n$-assemblies from which it is drawn. It will be shown that mixing (i.e. using assemblies issued from different distributions) improves performance by a factor, hereby called the mixing factor, of up to (but not including) 2. We will further show that when all similar $n$-assemblies have the same performance, the mixing factor is at least 1 .

While an extensive literature exists on the expected value of the maximum of $n$ independent and identically distributed random variables (see for example [3]), with the exemption of [1] and [6] (see also [2] and [3, Section 5.2]), not much work has concentrated on the case of non-identically distributed random variables. Furthermore, the aforementioned papers do not attempt a comparison with $M_{1}, \ldots, M_{n}$. In [1, the authors obtain upper and lower bounds in terms of $\mathbb{E}\left[X_{i}\right]$ and $\operatorname{var}\left(X_{i}\right)$ (assumed to be finite) where $X_{1}, \ldots, X_{n}$ are possibly dependent random variables with possibly different distributions. These bounds generalise those of [4] and [5] which deal with the independent and identically distributed case. In [6], the author solely focuses on obtaining a lower bound. This is done by comparing

Key words and phrases. mixing, stochastic ordering, distribution of the maximum AMS Classification: 60E15, 60K10. 
the distribution function of the maximum of $X_{1}, \ldots, X_{n}$ to that of the maximum of $n$ independent copies of an equally-weighted probability mixture of $X_{1}, \ldots, X_{n}$ (see later). More recently, in [7], the author investigates the performance of an $n$-assembly constructed from 2 distributions and its behaviour as the make-up of the $n$-assembly changes.

Notations and Assumptions. Throughout this paper, we use the following notations and assumptions. For any sequence $x_{1}, \ldots, x_{n}$, we write $x_{1} \vee x_{2} \vee \ldots \vee x_{n}=\bigvee_{k=1}^{n} x_{k}$ for the maximum $\max \left(x_{1}, x_{2}, \ldots, x_{n}\right)$. Random variables will generally be indexed in the following way: $X_{i}^{j}$ refers to the $j$ th element in the similar $n$-assembly $i$; i.e. $X_{i}^{1}, \ldots, X_{i}^{n}$ are independent random variables having the same distribution as $X_{i} . X_{i}^{(n)}=\bigvee_{k=1}^{n} X_{i}^{k}$, $X_{(n)}=\bigvee_{i=1}^{n} X_{i}, M_{i}=\mathbb{E}\left[X_{i}^{(n)}\right], M_{(n)}=\bigvee_{i=1}^{n} M_{i}$ and $\bar{M}=\frac{1}{n} \sum_{i=1}^{n} M_{i}$. Finally, we denote by $\theta_{n}$ the mixing factor of a set of $n$-assemblies:

$$
\theta_{n}=\frac{\mathbb{E}\left[X_{(n)}\right]}{M_{(n)}}
$$

We assume that all random variables are non-negative and have finite first moment.

\section{The Mixing Factor Bounds}

2.1. The Main Results. The main theorem which we wish to show is:

Theorem 1. Let $X_{1}, \ldots, X_{n}$ be independent random variables and $M_{i}=\mathbb{E}\left[X_{i}^{(n)}\right], i=$ $1,2, \ldots, n$. Then

$$
\bar{M} \leq \mathbb{E}\left[X_{(n)}\right] \leq \bar{M}+\frac{n-1}{n} M_{(n)} .
$$

In particular, if $M_{i}=M, i=1, \ldots, n$,

$$
M \leq \mathbb{E}\left[X_{(n)}\right] \leq(2-1 / n) M .
$$

Corollary 2. For $n$-assemblies, the mixing factor does not exceed $2-1 / n$ and, in the case of equally performing similar $n$-assemblies, it is at least 1.

In the case where some of the random variables $X_{1}, \ldots, X_{n}$ are identically distributed, an improved upper bound may be achieved. Such a bound follows immediately from Theorem 1 when all distributions are repeated an equal amount.

Corollary 3. Suppose the $n$-assembly $X_{1}, \ldots, X_{n}$ is made up of $k$ similar $m$-assemblies $(n=k m)$. Then

$$
\bar{M} \leq \mathbb{E}\left[X_{(n)}\right] \leq \bar{M}+\frac{k-1}{k} M_{(n)} .
$$

Remark 4. Let $F_{i}$ denote the distribution function of $X_{i}$ and $G_{i}=F_{i}^{n}$ be the distribution function of $X_{i}^{(n)}$. Then (1) can be rewritten as

$$
0 \leq \int_{0}^{\infty}(\bar{G}(s)-\tilde{G}(s)) d s \leq(1-1 / n) \max _{1 \leq i \leq n} \int_{0}^{\infty}\left(1-G_{i}(s)\right) d s,
$$

where $\bar{G}$ and $\tilde{G}$ are the arithmetic and geometric means, respectively, of the distribution functions $G_{1}, G_{2}, \ldots, G_{n}$.

Equation (2) provides an upper bound (the lower bound is a direct consequence of the arithmetic and geometric mean inequality) on the $L^{1}$-distance between the geometric and 
arithmetic means of a sequence of $n$ distribution functions on the positive half-line with finite means.

In probabilistic terms, this expresses the following fact. Let $Y_{1}, \ldots, Y_{n}$ be independent random variables (non-negative with finite mean). Let $U$ be an equally-weighted probability mixture of $Y_{1}, \ldots, Y_{n}$ and $V$ be such that the distribution of the maximum of $n$ independent copies of $V$ is that of $Y_{(n)}$. Then

$$
0 \leq \mathbb{E}[V]-\mathbb{E}[U] \leq(1-1 / n) \max _{1 \leq i \leq n} \mathbb{E}\left[Y_{i}\right]
$$

As hinted in the introduction, the lower bound is not optimal. In [6] (see also [2] and [3, Section 5.2]), it is shown that

$$
\mathbb{P}\left(X_{(n)} \leq x\right) \leq \mathbb{P}\left(Z^{(n)} \leq x\right), \quad \forall x>0,
$$

where $Z^{1}, \ldots, Z^{n}$ are independent copies of an equally-weighted probability mixture of $X_{1}, \ldots, X_{n}$. Combining the inequality of the arithmetic and geometric means with a moment inequality, we can further bound the right hand side of (3)

$$
\mathbb{P}\left(Z^{(n)} \leq x\right)=\left(\frac{1}{n} \sum_{k=1}^{n} \mathbb{P}\left(X_{k} \leq x\right)\right)^{n} \leq \frac{1}{n} \sum_{k=1}^{n} \mathbb{P}\left(X_{k} \leq x\right)^{n}=\frac{1}{n} \sum_{k=1}^{n} \mathbb{P}\left(X_{k}^{(n)} \leq x\right) .
$$

As a consequence, we obtain an improved lower bound for $\mathbb{E}\left[X_{(n)}\right]$,

$$
\bar{M} \leq \mathbb{E}\left[Z^{(n)}\right] \leq \mathbb{E}\left[X_{(n)}\right]
$$

Although a better bound for the performance of an $n$-assembly, $\mathbb{E}\left[Z^{(n)}\right]$ cannot be expressed in terms of the performances of all similar $n$-assemblies from which it is drawn. In other words, $\mathbb{E}\left[Z^{(n)}\right]$ is not a bound that can be expressed in terms of $M_{1}, \ldots, M_{n}$. Furthermore, it is shown in Corollary 13 that, in the case of bounded random variables, an improved lower bound, expressed in terms of $M_{1}, \ldots, M_{n}$, can be achieved. This lower bound is shown, in the special case of a two-point distribution, to outperform $\mathbb{E}\left[Z^{(n)}\right]$.

2.2. Two Toy Examples. Before we embark on the proof of the main Theorem, we look at the simple case of two-point distributions. The first example presented here will give some insight into how the improved bound of Corollary 13 and that of [6] compare. The second example will demonstrate the sharpness of the upper bound of Theorem 1; i.e. we show that $\mathbb{E}\left[X_{(n)}\right]$ can be made as close to the upper bound as we want.

(1) Assume that the random variables $X_{1}, \ldots, X_{n}$ are all concentrated on two points, 0 and $b$, and let $p_{i}=\mathbb{P}\left[X_{i}=0\right]$. Then the distribution of $Z$, the equally-weighted probability mixture of $X_{1}, \ldots, X_{n}$, is given by $\mathbb{P}[Z=0]=\frac{1}{n} \sum_{i=1}^{n} p_{i}=\bar{p}$ and,

$$
\mathbb{E}\left[Z^{(n)}\right]=b\left(1-\bar{p}^{n}\right) \text { and } M_{i}=b\left(1-p_{i}^{n}\right) .
$$

Therefore,

$$
b-\prod_{i=1}^{n} \sqrt[n]{b-M_{i}}-\mathbb{E}\left[Z^{(n)}\right]=b \bar{p}^{n}-b \prod_{i=1}^{n} p_{i} \geq 0,
$$

by a simple application of the inequality for the arithmetic and geometric means. 
(2) Again, we assume that the random variables $X_{1}, \ldots, X_{n}$ are all concentrated on two points, or less. However, in this case, we allow the non-zero values $x_{1}, \ldots, x_{n}$, to be different. In fact, we assume (without loss of generality) that $X_{n}$ is non-random, that all other random variables take 0 , with probability $p_{k}=\sqrt[n]{1-M_{k} / x_{k}}>0$, and $x_{k}$, with probability $1-p_{k}>0$, and that

$$
M_{(n-1)} \leq M_{n}<x_{1}<\ldots<x_{n-1}
$$

Then, it is shown in the proof of Proposition 8 (from which this very construction is extracted), that

$$
\mathbb{E}\left[X_{(n-1)} \vee M_{n}\right]=p_{n-1} \mathbb{E}\left[X_{(n-2)} \vee M_{n}\right]+\left(1-p_{n-1}\right) \frac{M_{n-1}}{1-p_{n-1}^{n}} .
$$

Letting $p_{n-1}$ go to 1 and $x_{n-1}$ to infinity so that $M_{n-1}$ remains constant, we see that $\mathbb{E}\left[X_{(n)}\right]$ approaches $\mathbb{E}\left[X_{(n-2)} \vee M_{n}\right]+\frac{M_{n-1}}{n}$. Now letting $p_{n-2}$ go to 1 and $x_{n-2}$ to infinity so that $M_{n-2}$ remains constant, we see that $\mathbb{E}\left[X_{(n)}\right]$ approaches $\mathbb{E}\left[X_{(n-3)} \vee M_{n}\right]+\frac{M_{n-2}+M_{n-1}}{n}$. Repeating this process leads to the fact that $\mathbb{E}\left[X_{(n)}\right]$ approaches $M_{n}+\frac{M_{1}+\ldots+M_{n-1}}{n}=\bar{M}+\frac{n-1}{n} M_{(n)}\left(\right.$ recall that $\left.M_{n}=M_{(n)}\right)$.

2.3. The Proofs. The lower bound is a direct consequence of the inequality for arithmetic and geometric means and is given here for completeness only.

Proposition 5. If $X_{1}, \ldots, X_{n}$ are independent random variables with the property that $\mathbb{E}\left[X_{i}^{(n)}\right]=M_{i}, i=1,2, \ldots, n$, then $\mathbb{E}\left[X_{(n)}\right] \geq \bar{M}$. Furthermore, if $\mathbb{E}\left[X_{(n)}\right]=\bar{M}$ then $X_{1}, \ldots, X_{n}$ are identically distributed.

Proof. Because the arithmetic mean is greater than the geometric mean,

$$
1-u_{1} u_{2} \ldots u_{n} \geq \frac{1}{n} \sum_{i=1}^{n}\left(1-u_{i}^{n}\right) .
$$

This implies

$$
\mathbb{E}\left[X_{(n)}\right]=\int_{0}^{\infty}\left[1-F_{1}(x) F_{2}(x) \ldots F_{n}(x)\right] d x \geq \frac{1}{n} \sum_{i=1}^{n} \int_{0}^{\infty}\left[1-F_{i}^{n}(x)\right] d x=\bar{M},
$$

where $F_{i}$ denotes the distribution function of $X_{i}$. Further, since (4) turns into an equality if and only if the $u_{i}$ 's are all equal, the lower bound of $\mathbb{E}\left[X_{(n)}\right]$ is only attained when $F_{1}=\ldots=F_{n}$, that is when $X_{1}, \ldots, X_{n}$ are identically distributed.

The first step in the proof of the upper bound is to reduce the problem to the case of random variables concentrated on a finite set of points. This is easily demonstrated by using the approximation

$$
X=\lim _{m \uparrow \infty}\left[\sum_{l=1}^{m 2^{m}} \frac{l-1}{2^{m}} 1_{\left[(l-1) / 2^{m}, l / 2^{m}\right)}(X)+m 1_{[m,+\infty)}(X)\right] .
$$

Indeed, one only needs to apply the Monotone Convergence Theorem to prove the following proposition. 
Proposition 6. If Theorem 1 is true for random variables concentrated on a finite set of points, then it must be true for general random variables.

Having reduced the problem to one that only involves random variables concentrated on a finite set of points, we shall find the maximum possible value of $\mathbb{E}\left[X_{(n)}\right]$ when we fix $\mathbb{E}\left[X_{i}^{(n)}\right]=M_{i}, i=1,2, \ldots, n$, by continually creating new sets of $n$-assemblies which maintain the property $\mathbb{E}\left[X_{i}^{(n)}\right]=M_{i}$, but increase, or at least do not decrease, $\mathbb{E}\left[X_{(n)}\right]$.

The main step in achieving the upper bound announced in Theorem 1 is to prove that the problem can be reduced to one that involves random variables that take exactly one non-zero value. This is done by showing that, for any random variable, two nonzero adjacent points can be coalesced into a single point; that is, a random variable which takes values $x_{1}, \ldots, x_{r}\left(x_{1}<\ldots<x_{r}\right)$ with probabilities $p_{1}, \ldots, p_{r}$ respectively, can be replaced by a random variable with masses $p_{1}, \ldots, p_{i-1}, p_{i}+p_{i+1}, p_{i+2}, \ldots, p_{r}$ at $x_{1}, \ldots, x_{i-1}, x, x_{i+2}, \ldots, x_{r}$, for a carefully chosen $x$. This is initially done in Proposition 9 for adjacent points not separated by points from other random variables, and later extended to the general case in Proposition 10 yielding the following Theorem.

Proposition 7. If Theorem 1 is true for random variables which take exactly one non-zero value, then it must be true for general random variables.

Proposition 8. Let $X_{1}, \ldots, X_{n}$ be independent random variables with the property that $\mathbb{E}\left[X_{i}^{(n)}\right]=M_{i}, i=1,2, \ldots, n$. If each of $X_{1}, \ldots, X_{n}$ takes exactly one non-zero value, then

$$
\bar{M} \leq \mathbb{E}\left[X_{(n)}\right] \leq \bar{M}+\frac{n-1}{n} M_{(n)} .
$$

Proof. For $k \in\{1, \ldots, n\}$, we denote by $x_{k}$ the non-zero value of $X_{k}$ and $p_{k}=\mathbb{P}\left[X_{k}=0\right]$. The next four points successively simplify the problem.

(1) If $\mathbb{P}\left[X_{k}=0\right]>0$, for all $k$ (i.e. all $X_{k}$ 's place a positive mass at 0 ), then applying Proposition 9 to $X_{1}$ (assumed without loss of generality to have the smallest nonzero value) and $Y=\bigvee_{k=2}^{n} X_{k}$ shows that $\mathbb{E}\left[X_{(n)}\right]$ is increased if $X_{1}$ is replaced by $M_{1}=\mathbb{E}\left[X_{1}^{(n)}\right]$. Therefore we may assume that at least one of the $X_{k}$ 's is constant.

(2) Without loss of generality, we may assume that $X_{n}$ is the largest constant random variable. $X_{n}$ may be one of any number of constant random variables, all equal, that are larger than any other constant random variable. Assume that at least one constant random variable is strictly smaller than $X_{n}$. Let it be $a\left(a<X_{n}\right)$. Since $\mathbb{E}\left[a \vee X_{n} \vee Y\right]=\mathbb{E}\left[Z \vee X_{n} \vee Y\right]$, where $Z$ is such that $\mathbb{P}[Z=0]=\left(1-a / X_{n}\right)^{1 / n}$ and $\mathbb{P}\left[Z=X_{n}\right]=1-\left(1-a / X_{n}\right)^{1 / n}\left(a \vee X_{n}=X \vee X_{n}=X_{n}\right)$, we may assume that all $X_{k}$ 's satisfying $M_{k}<M_{n}$, are non-constant (place a positive mass on 0 ).

(3) If one other $X_{k}$, say $X_{n-1}$, satisfies $X_{n-1}=M_{n-1}=M_{n}$, then replacing $X_{n-1}$ by $Y$ such that $\mathbb{P}[Y=0]=q>0, \mathbb{P}[Y=y]=1-q$ and $y=M_{n} /\left(1-q^{n}\right)>M_{n}$, we get

$$
\begin{aligned}
& \mathbb{E}\left[X_{(n-2)} \vee Y \vee M_{n}\right] \\
& \quad=q \mathbb{E}\left[X_{(n-2)} \vee M_{n}\right]+(1-q) \mathbb{E}\left[X_{(n-2)} \vee y \vee M_{n}\right] \\
& \quad \geq \mathbb{E}\left[X_{(n-2)} \vee M_{n}\right]=\mathbb{E}\left[X_{(n)}\right] .
\end{aligned}
$$

Therefore we may assume that the random variable with the largest $M_{k}$ (say $M_{n}$ ) is constant and all others are non-constant. 
(4) Without loss of generality, we may assume that $x_{1} \leq \ldots \leq x_{n-1}$. Recall that, for $k \in\{1, \ldots, n-1\}, M_{k}=\left(1-p_{k}^{n}\right) x_{k}$. If $x_{1} \leq M_{n}$ then, with $Y$ such that $\mathbb{P}[Y=0]=q>0, \mathbb{P}[Y=y]=1-q$ and $y=M_{1} /\left(1-q^{n}\right)>M_{n}$,

$$
\mathbb{E}\left[Y \vee Z \vee M_{n}\right]=q \mathbb{E}\left[Z \vee M_{n}\right]+(1-q) \mathbb{E}\left[y \vee Z \vee M_{n}\right]>\mathbb{E}\left[X_{(n)}\right],
$$

where $Z=\bigvee_{k=2}^{n-1} X_{k}$. Therefore we may assume that $M_{(n-1)} \leq M_{n}<x_{1} \leq \ldots \leq$ $x_{n-1}$.

Now,

$$
\begin{aligned}
\mathbb{E}\left[X_{(n)}\right] & =\mathbb{E}\left[X_{(n-2)} \vee X_{n-1} \vee M_{n}\right] \\
& =p_{n-1} \mathbb{E}\left[X_{(n-2)} \vee M_{n}\right]+\left(1-p_{n-1}\right) x_{n-1} \\
& =p_{n-1} \mathbb{E}\left[X_{(n-2)} \vee M_{n}\right]+\left(1-p_{n-1}\right) \frac{M_{n-1}}{1-p_{n-1}^{n}}
\end{aligned}
$$

Next we use the fact that the function $\phi(p)=u p+v \frac{1-p}{1-p^{n}}$ defined on $[0,1]$ (extended at 1 by continuity), where $v<u$, increases from $v$ to $u+v / n$. Since $\mathbb{E}\left[X_{(n-2)} \vee M_{n}\right]>M_{n-1}$, we immediately get that

$$
\mathbb{E}\left[X_{(n)}\right] \leq \mathbb{E}\left[X_{(n-2)} \vee M_{n}\right]+\frac{M_{n-1}}{n} .
$$

Repeating the same argument, we get

$$
\mathbb{E}\left[X_{(n)}\right] \leq \mathbb{E}\left[X_{(n-3)} \vee M_{n}\right]+\frac{M_{n-2}+M_{n-1}}{n} \leq \ldots \leq M_{n}+\frac{M_{1}+\ldots+M_{n-1}}{n} .
$$

\section{Comparison Results}

In this section we develop the tools required to obtain the bounds of the previous section. However, these tools are important in their own right. They enable us to increase the performance of an $n$-assembly while keeping the performances of all similar $n$-assemblies unchanged. We prove them for random variables that are not necessarily concentrated on a finite set of points. We also obtain a comparison result that allows to decrease the performance of an $n$-assembly while keeping the performances of all similar $n$-assemblies unchanged.

Proposition 9. If $X_{1}$ and $Y$ are independent, $\mathbb{P}\left[a \leq X_{1} \leq b\right]=p$ and $\mathbb{P}[a<Y<b]=0$ then we may replace $X_{1}$ by a random variable $X_{2}$ such that $X_{2}=X_{1}$ outside $[a, b]$ and, $\mathbb{P}\left[a \leq X_{2} \leq b\right]=\mathbb{P}\left[X_{2}=x\right]=p$, for some $a<x<b$ with the property that $\mathbb{E}\left[X_{2}^{(n)}\right]=$ $\mathbb{E}\left[X_{1}^{(n)}\right]$ and $\mathbb{E}\left[X_{2} \vee Y\right] \geq \mathbb{E}\left[X_{1} \vee Y\right]$

Proof. Equating the contributions to $\mathbb{E}\left[X_{1}^{(n)}\right]$ and $\mathbb{E}\left[X_{2}^{(n)}\right]$ from the interval $[a, b]$ gives

$$
x\left(F(b)^{n}-F^{-}(a)^{n}\right)=\sum_{k=1}^{n}\left(\begin{array}{l}
n \\
k
\end{array}\right) p^{k} F^{-}(a)^{n-k} \mathbb{E}\left[X_{1}^{(k)} \mid a \leq X_{1}^{j} \leq b, j=1, \ldots, k\right]
$$

where $F$ is the distribution function of $X_{1}$ and $F^{-}(x)=\lim _{y \uparrow x} F(y)$. We thus see that $x$ is a convex combination of the $n$ expectations on the right hand side and thus, in particular, that $x \geq \min _{1 \leq k \leq n} \mathbb{E}\left[X_{1}^{(k)} \mid a \leq X_{1} \leq b\right]=\mathbb{E}\left[X_{1} \mid a \leq X_{1} \leq b\right]$. Finally

$$
\mathbb{E}\left[X_{2} \vee Y\right]-\mathbb{E}\left[X_{1} \vee Y\right]=p \mathbb{P}[Y<a]\left(x-\mathbb{E}\left[X_{1} \mid a \leq X_{1} \leq b\right]\right)
$$


and, as previously observed, the second expression is nonnegative.

If we allow $\mathbb{P}[a<Y<b]>0$ then the coalescing point is no longer necessarily in the interval $[a, b]$ as is demonstrated in the next result.

Proposition 10. Assume that $X_{1}$ and $Y$ are independent and that the entire mass $X_{1}$ places on an interval $(l, r)$ is concentrated on two values $a$ and $b$ within it: $0 \leq l<a<$ $b<r, \mathbb{P}\left[l<X_{1}<r\right]=\mathbb{P}\left[X_{1} \in\{a, b\}\right], p=\mathbb{P}\left[X_{1}=a\right]>0$ and $q=\mathbb{P}\left[X_{1}=b\right]>0$. Then there exists a random variable $X_{2}$ s.t. $\mathbb{E}\left[X_{1}^{(n)}\right]=\mathbb{E}\left[X_{2}^{(n)}\right], \mathbb{E}\left[X_{1} \vee Y\right]<\mathbb{E}\left[X_{2} \vee Y\right]$ and the mass $X_{2}$ places on the interval $(l, r)$ is concentrated on at most one single value within it.

Proof. Let $X_{2}=X_{1} 1_{X_{1} \leq l}+u 1_{X_{1}=a}+V(u) 1_{X_{1}=b}+X_{1} 1_{X_{1} \geq r}$, where $u$ is a free parameter,

$$
V(u)=b-\lambda_{n}(u-a) \text { and } \lambda_{n}=\frac{F(a)^{n}-F(l)^{n}}{F(b)^{n}-F(a)^{n}} .
$$

Then $\mathbb{E}\left[X_{2}^{(n)}\right]=\mathbb{E}\left[X_{1}^{(n)}\right]$ and $\mathbb{E}\left[X_{2} \vee Y\right]=\mathbb{E}\left[X_{1} \vee Y\right]+\phi(u)-\phi(a)$, where

$$
\phi(u)=p \mathbb{E}[u \vee Y]+q \mathbb{E}[V(u) \vee Y] .
$$

Using Lemma 11 below, we get that $\phi_{+}^{\prime}(u)=p G(u)-q \lambda_{n} G_{-}(V(u))$ is non-decreasing and that $\phi_{+}^{\prime}(a)=p G(a)-q \lambda_{n} G_{-}(b)$ is not zero unless $G(a)>0$ (since $G_{-}(b) \geq \mathbb{P}[a<Y<$ $b]>0)$.

Therefore there are three possible scenarios, either $\phi_{+}^{\prime}(a)<0, \phi_{+}^{\prime}(a)>0$ or, $\phi_{+}^{\prime}(a)=0$ and $G(a)>0$. In the first case, $\mathbb{E}\left[X_{2} \vee Y\right]$ decreases on $[l, a]$ and letting $u=l$ achieves the objective. In the second case, $\mathbb{E}\left[X_{2} \vee Y\right]$ increases on $[a, r]$ and it suffices to let $u=r$.

Finally, suppose $\phi_{+}^{\prime}(a)=0$ and $G(a)>0$. Let $\omega=\mathbb{E}\left[X_{1}^{(n)} \mid l<X_{1}^{(n)}<r\right]$. Then $a<\omega<b$, $V(\omega)=\omega$ and

$$
\phi_{+}^{\prime}(\omega) \geq G(\omega) \frac{p q}{F(b)^{n}-F(a)^{n}} \sum_{k=0}^{n-1} F(a)^{k}\left[F(b)^{n-k-1}-F(l)^{n-k-1}\right]>0 .
$$

Here it suffices to let $u=\omega(a$ and $b$ are merged into $\omega)$.

Lemma 11. For any positive random variable $Y$ with distribution function $G$ and differentiable function $h, \gamma(u)=\mathbb{E}[h(u) \vee Y]$ admits left and right derivatives (that differ on a set that is at most countable):

$$
\gamma_{ \pm}^{\prime}(u)=\left\{\begin{array}{lll}
h^{\prime}(u) G_{ \pm}(h(u)) & \text { if } \quad h^{\prime}(u) \geq 0 \\
h^{\prime}(u) G_{\mp}(h(u)) & \text { if } \quad h^{\prime}(u)<0
\end{array}\right.
$$

The next proposition enables the reduction of $\mathbb{E}\left[X_{(n)}\right]$ for given $M_{i}, i=1, \ldots, n$.

Proposition 12. Let $b$ be such that $\mathbb{P}\left[X_{i} \leq b\right]>0$, for all $i$. Then, for any interval $I=[a, b]$, there are random variables $Y_{1}, \ldots, Y_{n}$ such that $\mathbb{E}\left[X_{(n)}\right] \geq \mathbb{E}\left[Y_{(n)}\right]$ and, for all $i=1, \ldots, n, Y_{i}=X_{i}$ on $\left\{X_{i} \notin I\right\}, \mathbb{P}\left[a<Y_{i}<b\right]=0$ and $\mathbb{E}\left[X_{i}^{(n)}\right]=\mathbb{E}\left[Y_{i}^{(n)}\right]$.

Proof. Let $\xi_{i}, i=1, \ldots, n$, be such that $\mathbb{P}\left[\xi_{i}=a\right]=\alpha_{i}, \mathbb{P}\left[\xi_{i}=b\right]=1-\alpha_{i}$ and $\xi_{1}, \ldots, \xi_{n}$ are independent of each other and of all other random variables $X_{i}$. Now let

$$
Y_{i}=\xi_{i} 1_{X_{i} \in I}+X_{i} 1_{X_{i} \notin I}
$$


and form the corresponding similar $n$-assemblies, $\left(Y_{i}^{1}, \ldots, Y_{i}^{n}\right)_{i=1, \ldots, n}$. Our first objective is to select $\alpha_{i}$ such that $\mathbb{E}\left[X_{i}^{(n)}\right]=\mathbb{E}\left[Y_{i}^{(n)}\right]$. For simplicity, we shall momentarily drop the index $i$ and compute more generally $\mathbb{E}\left[X_{(n)}\right]-\mathbb{E}\left[Y_{(n)}\right]$. Further, we introduce the notation, $\hat{z}=z \vee a-a=(z-a)^{+}$and observe that,

(1) $a \leq z \leq b$ iff $z \geq a$ and $\hat{z} \leq b-a$

(2) $\widehat{z_{(n)}}=\hat{z}_{(n)}$

(3) if $\mathcal{K}$ is set of non-empty subsets of $N=\{1, \ldots, n\}$,

$$
\hat{z}_{(n)} \prod_{k=1}^{n} 1_{z_{k} \geq a}=\hat{z}_{(n)}-\sum_{K \in \mathcal{K}}\left(\bigvee_{k \in K} \hat{z}_{k}\right)\left(\prod_{k \in K} 1_{z_{k} \in I}\right)\left(\prod_{k \notin K} 1_{z_{k}<a}\right) .
$$

For $K \in \mathcal{K}$, let

$$
A_{K}=\bigcap_{k \in K}\left\{X_{k} \in I\right\}, B_{K}=\bigcap_{k \notin K}\left\{X_{k}<a\right\}, C_{K}=A_{K} \cap B_{K}
$$

and for any sequence $z_{1}, \ldots, z_{n}, z_{[K]}=\bigvee_{k \in K} z_{k}$. Using the identities,

$$
\left\{X_{(n)} \in I\right\}=\bigcup_{K \in \mathcal{K}} C_{K} \text { and } X_{(n)}-Y_{(n)}=\sum_{K \in \mathcal{K}}\left(X_{[K]}-\xi_{[K]}\right) 1_{C_{K}},
$$

we obtain

$$
\begin{aligned}
\mathbb{E}\left[X_{(n)}\right]-\mathbb{E}\left[Y_{(n)}\right] \\
=\sum_{K \in \mathcal{K}}\left(\mathbb{E}\left[X_{[K]}, C_{K}\right]-\mathbb{E}\left[\xi_{[K]}, C_{K}\right]\right) \\
=\sum_{K \in \mathcal{K}}\left(\mathbb{E}\left[\hat{X}_{[K]}+a, C_{K}\right]-\mathbb{E}\left[\hat{\xi}_{[K]}+a, C_{K}\right]\right) \\
=\sum_{K \in \mathcal{K}}\left(\mathbb{E}\left[\hat{X}_{[K]}, C_{K}\right]-\mathbb{E}\left[\hat{\xi}_{[K]}, C_{K}\right]\right) \\
=\sum_{K \in \mathcal{K}} \mathbb{P}\left(B_{K}\right)\left(\mathbb{E}\left[\hat{X}_{[K]}, A_{K}\right]-\mathbb{E}\left[\hat{\xi}_{[K]}, A_{K}\right]\right) \\
=\quad \mathbb{E}\left[\hat{X}_{(n)}, A_{N}\right]+\sum_{K \in \mathcal{K} \backslash\{N\}} \mathbb{P}\left(B_{K}\right) \mathbb{E}\left[\hat{X}_{[K]}, A_{K}\right]-\sum_{K \in \mathcal{K}} \mathbb{P}\left(A_{K}\right) \mathbb{P}\left(B_{K}\right) \mathbb{E}\left[\hat{\xi}_{[K]}\right] \\
=\quad \mathbb{E}\left[\hat{X}_{(n)}, D_{N}\right]-\sum_{K \in \mathcal{K} \backslash\{N\}} \mathbb{E}\left[\hat{X}_{[K]}, D_{N} \cap B_{K} \cap E_{K}\right] \\
\quad+\sum_{K \in \mathcal{K} \backslash\{N\}} \mathbb{P}\left(B_{K}\right) \mathbb{E}\left[\hat{X}_{[K]}, A_{K}\right]-\sum_{K \in \mathcal{K}} \mathbb{P}\left(A_{K}\right) \mathbb{P}\left(B_{K}\right) \mathbb{E}\left[\hat{\xi}_{[K]}\right]
\end{aligned}
$$


where $D_{K}=\bigcap_{k \in K}\left\{\hat{X}_{k} \leq b-a\right\}, E_{K}=\bigcap_{k \in K}\left\{X_{k} \geq a\right\}$ and we have used (5). Applying the identities $D_{N} \cap B_{K}=D_{K} \cap B_{K}$ and $D_{K} \cap E_{K}=A_{K}$, it follows that

$$
\begin{aligned}
& \mathbb{E}\left[X_{(n)}\right]-\mathbb{E}\left[Y_{(n)}\right] \\
& =\mathbb{E}\left[\hat{X}_{(n)}, D_{N}\right]-\sum_{K \in \mathcal{K} \backslash\{N\}} \mathbb{P}\left(B_{K}\right) \mathbb{E}\left[\hat{X}_{[K]}, A_{K}\right] \\
& \quad+\sum_{K \in \mathcal{K} \backslash\{N\}} \mathbb{P}\left(B_{K}\right) \mathbb{E}\left[\hat{X}_{[K]}, A_{K}\right]-\sum_{K \in \mathcal{K}} \mathbb{P}\left(A_{K}\right) \mathbb{P}\left(B_{K}\right) \mathbb{E}\left[\hat{\xi}_{[K]}\right] \\
& =\mathbb{E}\left[\hat{X}_{(n)}, D_{N}\right]-\sum_{K \in \mathcal{K}} \mathbb{P}\left(A_{K}\right) \mathbb{P}\left(B_{K}\right) \mathbb{E}\left[\hat{\xi}_{[K]}\right]
\end{aligned}
$$

Therefore, with $p_{i}=\mathbb{P}\left[X_{i} \in I\right], \mathbb{E}\left[X_{i}^{(n)}\right]=\mathbb{E}\left[Y_{i}^{(n)}\right]$ if and only if

$$
\begin{aligned}
\mathbb{E}\left[\hat{X}_{i}^{(n)}, \hat{X}_{i}^{(n)} \leq b-a\right] & =\sum_{m=1}^{n}\left(\begin{array}{c}
n \\
m
\end{array}\right) p_{i}^{m} F_{i}^{-}(a)^{n-m}\left(1-\alpha_{i}^{m}\right)(b-a) \\
& =(b-a)\left[\sum_{m=1}^{n}\left(\begin{array}{c}
n \\
m
\end{array}\right) p_{i}^{m} F_{i}^{-}(a)^{n-m}-\sum_{m=1}^{n}\left(\begin{array}{c}
n \\
m
\end{array}\right) p_{i}^{m} \alpha_{i}^{m} F_{i}^{-}(a)^{n-m}\right] \\
& =(b-a)\left[F_{i}(b)^{n}-\left(F_{i}^{-}(a)+p_{i} \alpha_{i}\right)^{n}\right]
\end{aligned}
$$

that is

$$
\left(\frac{F_{i}^{-}(a)+p_{i} \alpha_{i}}{F_{i}(b)}\right)^{n}(b-a)=(b-a)-\mathbb{E}\left[\hat{X}_{i}^{(n)} \mid \hat{X}_{i}^{(n)} \leq b-a\right]
$$

Returning to (6), we find

$$
\begin{aligned}
\mathbb{E}\left[X_{(n)}\right]-\mathbb{E}\left[Y_{(n)}\right] & \mathbb{E}\left[\hat{X}_{(n)}, D_{N}\right]-\sum_{K \in \mathcal{K}} \mathbb{P}\left(A_{K}\right) \mathbb{P}\left(B_{K}\right) \mathbb{E}\left[\hat{\xi}_{[K]}\right] \\
= & \mathbb{E}\left[\hat{X}_{(n)}, \hat{X}_{(n)} \leq b-a\right]-\sum_{K \in \mathcal{K}} \prod_{k \in K} p_{k} \prod_{k \notin K} F_{k}^{-}(a)\left(1-\prod_{k \in K} \alpha_{k}\right)(b-a) \\
= & \mathbb{E}\left[\hat{X}_{(n)} \mid \hat{X}_{(n)} \leq b-a\right] \prod_{k=1}^{n} F_{k}(b) \\
& -\left[\prod_{k=1}^{n} F_{k}(b)-\prod_{k=1}^{n} F_{k}^{-}(a)-\prod_{k=1}^{n}\left(F_{k}^{-}(a)+p_{k} \alpha_{k}\right)+\prod_{k=1}^{n} F_{k}^{-}(a)\right](b-a) \\
= & \mathbb{E}\left[\hat{X}_{(n)} \mid \hat{X}_{(n)} \leq b-a\right] \prod_{k=1}^{n} F_{k}(b)-\left[\prod_{k=1}^{n} F_{k}(b)-\prod_{k=1}^{n}\left(F_{k}^{-}(a)+p_{k} \alpha_{k}\right)\right](b-a) \\
= & \prod_{k=1}^{n} F_{k}(b)\left\{\mathbb{E}\left[\hat{X}_{(n)} \mid \hat{X}_{(n)} \leq b-a\right]-(b-a)+(b-a) \prod_{k=1}^{n} \frac{F_{k}^{-}(a)+p_{k} \alpha_{k}}{F_{k}(b)}\right\} .
\end{aligned}
$$


Now, by a simple application of Hölder's inequality, we get

$$
\begin{aligned}
\left((b-a)-\mathbb{E}\left[\hat{X}_{(n)} \mid \hat{X}_{(n)} \leq b-a\right]\right)^{n} & =\left(\int_{0}^{b-a} \prod_{k=1}^{n}\left(1-G_{k}(z)\right) d z\right)^{n} \\
& \leq \prod_{k=1}^{n} \int_{0}^{b-a}\left(1-G_{k}(z)\right)^{n} d z \\
& =\prod_{k=1}^{n}\left((b-a)-\mathbb{E}\left[\hat{X}_{k}^{(n)} \mid \hat{X}_{k}^{(n)} \leq b-a\right]\right) \\
& =(b-a)^{n} \prod_{k=1}^{n}\left(\frac{F_{k}^{-}(a)+p_{k} \alpha_{k}}{F_{k}(b)}\right)^{n}
\end{aligned}
$$

where $G_{k}$ is the conditional distribution function of $(b-a)-\hat{X}_{k}$ given $\left\{\hat{X}_{k} \leq b-a\right\}$ and we have used (7). It immediately follows (recall that $(b-a)-\mathbb{E}\left[\hat{X}_{k}^{(n)} \mid \hat{X}_{k}^{(n)} \leq b-a\right] \geq 0$ ) that

$$
(b-a)-\mathbb{E}\left[\hat{X}_{(n)} \mid \hat{X}_{(n)} \leq b-a\right] \leq(b-a) \prod_{k=1}^{n}\left(\frac{F_{k}^{-}(a)+p_{k} \alpha_{k}}{F_{k}(b)}\right)
$$

which completes the proof.

A consequence of this proposition is an improved lower bound in the case of bounded random variables.

Corollary 13. If $X_{1}, \ldots, X_{n}$ are independent bounded random variables with the property that $\mathbb{E}\left[X_{i}^{(n)}\right]=M_{i}, i=1,2, \ldots, n$, then

$$
\mathbb{E}\left[X_{(n)}\right] \geq b-\prod_{i=1}^{n} \sqrt[n]{b-M_{i}} \geq \bar{M}
$$

where $b$ is a common upper bound to all $X_{i}$ 's.

Proof. Applying Proposition 12 to the interval $I=[0, b]$ and the random variables $Y_{i}$ such that $\mathbb{P}\left[Y_{i}=0\right]=1-\mathbb{P}\left[Y_{i}=b\right]=\sqrt[n]{1-\frac{M_{i}}{b}}$, we immediately get that

$$
\mathbb{E}\left[X_{(n)}\right] \geq \mathbb{E}\left[Y_{(n)}\right]=\left(1-\prod_{i=1}^{n} \sqrt[n]{1-\frac{M_{i}}{b}}\right) b=b-\prod_{i=1}^{n} \sqrt[n]{b-M_{i}} \geq \bar{M}
$$

The second inequality is a direct consequence of the inequality for arithmetic and geometric means.

Acknowledgement. This research was supported by the Australian Research Council. The authors would also like to thank the anonymous referees for their most valuable comments, in particular about the existing literature.

\section{REFERENCES}

[1] Arnold B.C. and Groeneveld R.A. (1979), Bounds on Expectations of Linear Systematic Statistics Based on Dependent Samples, Ann. Stat. 7, pp 220-223..

[2] Balakrishnan N. and Balasubramanian K. (2008), Revisiting Sen's inequalities on order statistics, Statist. Probab. Letters 78, pp 616-621. 
[3] David H.A. and Nagaraja H.N. (2003), Order Statistics, John Wiley \& Sons, Hoboken, New Jersey, 3rd ed.

[4] Hartley H.O. and David H.A. (1954), Universal Bounds for Mean Range and Extreme Observation, Ann. Math. Stat. 25, pp 85-89..

[5] Gumbel E.J. (1954), The Maxima of the Mean Largest Value and of the Range, Ann. Math. Stat. 25, pp 76-84..

[6] Sen P.K. (1970), A Note on Order Statistics for Heterogeneous Distributions, Ann. Math. Stat. 41, pp 2137-2139..

[7] Tokarev D. (2007), Galton-Watson processes and extinction in population systems, PhD Thesis, Monash University.

Kais Hamza, School of Mathematical Sciences, Monash University

Peter Jagers, Mathematical Statistics, Chalmers University of Technology

Aidan Sudbury, School of Mathematical Sciences, Monash University

Daniel Tokarev, School of Mathematical Sciences, Monash University 